\title{
Adapting to the impact of the COVID-19 pandemic on a randomized controlled trial examining lifestyle behaviors on cognitive functioning in obese African American adults: Building Research in Diet and Cognition (BRIDGE)
}

Marian Fitzgibbon ( $\nabla$ mlf@uic.edu )

University of Illinois at Chicago College of Medicine

Lara Blumstein

University of Illinois at Chicago

Linda Schiffer

University of Illinois at Chicago

Mirjana Antonic

University of Illinois at Chicago

Andrew McLeod

University of Illinois at Chicago

Roxanne Dakers

University of Illinois at Chicago

Leo Restrepo

University of Illinois at Chicago

Elizabeth Boots

University of Illinois at Chicago

Jennifer Sanchez-Flack

University of Illinois at Chicago

Lisa Tussing-Humphreys

University of Illinois at Chicago

Melissa Lamar

Rush University

Research Article

Keywords: Clinical Trials, Expedited Amendment Process, Remove Data Collection, Mediterranean Diet, Dietary Intake 
Posted Date: April 26th, 2021

DOl: https://doi.org/10.21203/rs.3.rs-290482/v1

License: (c) (i) This work is licensed under a Creative Commons Attribution 4.0 International License. Read Full License

Version of Record: A version of this preprint was published at Trials on July 16th, 2021. See the published version at https://doi.org/10.1186/s13063-021-05383-6. 
Adapting to the impact of the COVID-19 pandemic on a randomized controlled trial examining lifestyle behaviors on cognitive functioning in obese African American adults: Building Research in Diet and Cognition (BRIDGE)

Authors: Marian Fitzgibbon, PhD, ${ }^{a}, b, c, d$ Lara Blumstein, BA, ${ }^{a}$ Linda Schiffer, MS, ${ }^{a}$ Mirjana A. Antonic, M.Ed, ${ }^{a}$ Andrew McLeod, MS, ${ }^{a}$, e Roxanne Dakers, MPH, ${ }^{a}$ Leo Restrepo, MS, ${ }^{a}$ Elizabeth A. Boots, MS, ${ }^{a}$ Jennifer C. Sanchez-Flack, PhD, , ${ }^{\text {a }, ~ d}$ Lisa Tussing-Humphreys, PhD, ${ }^{\text {a, }}$, e and Melissa Lamar, $\mathrm{PhD}^{\mathrm{f}}$

a. Institute for Health Research and Policy, University of Illinois at Chicago, 1747 West Roosevelt Road, Chicago, IL 60608.

b. Department of Pediatrics, University of Illinois at Chicago, 1200 West Harrison Street, Chicago, IL 60607.

c. School of Public Health, University of Illinois at Chicago, 1603 W Taylor St, Chicago, IL 60612.

d. University of Illinois Cancer Center, University of Illinois at Chicago, 818 South Wolcott Avenue, Chicago, IL 60612.

e. Department of Kinesiology, University of Illinois at Chicago, 1919 W. Taylor St, Chicago, IL 60612.

f. Rush Alzheimer's Disease Center, Rush University, 1750 West Harrison Street, Chicago, IL 60612.

Corresponding Author:

Marian L. Fitzgibbon, PhD

1747 West Roosevelt Rd.

Chicago, IL 60608

mlf@uic.edu 


\section{Abstract}

Background. The COVID-19 pandemic has significantly altered the research landscape for clinical trials, requiring thoughtful consideration regarding how to handle the risks and benefits of continuing them.

Design. This brief report describes the experience of adapting the Building Research in Diet and Cognition (BRIDGE) study, a randomized clinical trial examining the effects of the Mediterranean Diet, with and without weight loss, on cognitive functioning in 185 older obese African American adults during the COVID-19 pandemic.

Measurement. The University of Illinois at Chicago (UIC) developed an expedited amendment process for research shifting to remote data collection. For the Cohort 3, 14-month data collection period, we adapted our protocol to allow data collection via telephone and e-mail. We were unable to collect certain measures that required face-to face contact.

Results. For measures that could be collected remotely, 14-month retention was similar for Cohort 3 and earlier cohorts: data were collected for $86.9 \%$ of cohort 3 (remote) and $87.9 \%$ of cohorts 1 and2 (face to face), $p=.84$.

Conclusions. In order to preserve the integrity of our clinical trial and ensure the safety of our participants and staff during the COVID-19 pandemic, we had to carefully and efficiently adapt our data collection procedures. The procedures put in place allowed us to collect our primary outcomes and the majority of our secondary outcomes and will enable us to examine the role of dietary intake, with and without weight loss, on cognitive functioning in a vulnerable and highrisk population.

ClinicalTrials.gov NCT03129048 


\section{Declarations}

Ethical Approval and Consent to participate: The BRIDGE Trial was approved by the University of Illinois at Chicago (UIC) Institutional Review Board. The trial is registered at

ClinicalTrials.gov (NCT3129048).

Consent for publication: Not Applicable

Availability of data and materials: The datasets used and/or analysed during the current study are available from the corresponding author on reasonable request.

Competing interests: The authors declare that they have no competing interests.

Funding: National Heart Lung and Blood Institute.

Authors' contributions:

MF - conception and design and drafted the manuscript

LB - substantial contributions to the conception and methods

LS - conducted the analyses and interpretation of data

MA - substantial contribution to revisions

$\mathrm{AM}$ - design and contribution to revisions

$\mathrm{RD}$ - contribution to methods

LR - contribution to methods

EB - contribution to methods

JSF - substantively revised the manuscript

LTH - substantively revised the manuscript

$\mathrm{ML}$ - contributed to design and substantively revised the manuscript

Acknowledgements: The authors would like to acknowledge study participants for giving generously of their time and effort on this study. We would also like to thank John Healy, General Manager at Columbus Vegetable Oils, and Judy Scott-McKay of the Almond Board of California, for their contribution of olive oil and almonds, respectively, to the study. This work was supported by the National Heart Lung and Blood Institute of the National Institutes of Health [R01HL129153].

Authors' information:

Marian Fitzgibbon, PhD: mlf@uic.edu

Lara Blumstein, BA: lbb@uic.edu

Linda Schiffer, MS: Ischiff@uic.edu 
Mirjana A. Antonic, M.Ed: manton6@uic.edu

Andrew McLeod, MS: amcleo2@uic.edu

Roxanne Dakers, MPH: rdakers@uic.edu

Leo Restrepo, MS: leo1724@uic.edu

Elizabeth A. Boots, MS: eboots2@uic.edu

Jennifer C. Sanchez-Flack, PhD: jsanch38@uic.edu

Lisa Tussing-Humphreys, PhD: Itussing@uic.edu

Melissa Lamar, PhD: melissa lamar@rusch.edu 


\section{INTRODUCTION}

The COVID-19 pandemic has dramatically changed how we work, socialize, and function on a day-to-day basis. At academic medical centers across the United States (US), the COVID-19 pandemic has altered many rigorously planned research protocols of randomized clinical trials (RCTs) ${ }^{1,2}$. At the time of this writing, there are 364,274 registered studies listed on ClinicalTrials.gov ${ }^{3}$. This includes 156,181 drug or biologic, 66,769 surgical or device, and 93,750 behavioral trials ${ }^{3}$. Also, at the time of this writing, this worldwide pandemic has infected more than $25,456,670$ and killed more than 427,626 people in the US ${ }^{4}$. It was apparent that to preserve our trial's integrity, we needed to quickly adapt our data collection procedures given the final data collection period of our three-arm RCT was scheduled to begin in April-May of 2020 , and we were unable to collect data in-person.

Briefly, the Building Research in Diet and Cognition (BRIDGE) trial is a three-arm RCT with 185 obese African American older adults (55 - 85 years of age) randomized to: 1) a Typical Diet Control; 2) a Mediterranean Diet lifestyle intervention without caloric restriction (MedDiet-A); or 3) a Mediterranean Diet with caloric restriction to promote weight loss (MedDiet-WL). The trial was designed to test whether MedDiet-WL would produce better results than a MedDiet-A on the primary outcome of cognitive functioning and secondary outcomes of body weight, body composition, dietary intake, lifestyle behaviors, and cardiometabolic-related biomarkers. Both MedDiet lifestyle interventions were 14-months long, including an 8-month, 27-session group intervention, followed by a 6 -month minimal contact period. The design and baseline characteristics of the sample are described in detail elsewhere ${ }^{5,6}$.

Before beginning our final data collection period for our third and final cohort, many national organizations such as the National Institutes of Health $(\mathrm{NIH})$, the Centers for Disease Control and Prevention (CDC), and the Office of Research and Development (ORD), had indicated research visits to be nonessential 7,8 . For trials funded through the $\mathrm{NIH}$, it was advised that 
investigators work with their respective Institutional Review Boards (IRBs) to assess the appropriate measures in order to guarantee the protection of both research participants and study staff. At the BRIDGE study site, the University of Illinois at Chicago (UIC) IRB instituted a hold on human subject research, including in-person, face-to-face interaction that was not critical from a patient care perspective. The IRB instructions, however, did indicate that research that could be conducted remotely was potentially acceptable.

The purpose of this paper is to document our experience of adapting the BRIDGE research protocol during the COVID-19 pandemic to meet data collection requirements, retention expectations, and ultimately, to answer our research questions. The BRIDGE trial is registered at ClinicalTrials.gov (NCT03129048).

\section{METHODS}

The BRIDGE Trial was conducted in three cohorts of approximately 60 persons each ${ }^{5}$. Participants provided data at baseline, immediately after the 8-month intervention, and at 14 months. Data collection assessed sociodemographic status, cognition, body weight, body composition, dietary intake, physical activity, cardiometabolic-related biomarkers, and health history. Prior to the pandemic onset, we were on course to begin our 14-month follow-up data collection for cohort 3 starting in April-May 2020.

The primary outcome of the study was change in cognitive functioning from baseline to post 8month intervention. The cognitive assessment protocol was a 60-minute neuropsychological protocol consistent with that developed by the National Institute of Neurological Disorders and Stroke and the Stroke-Canadian Networks Neuropsychology working group ${ }^{9}$. The secondary outcomes were related to body weight measured using a digital scale (Tanita, Arlington Heights, IL), whole-body composition using the General Electric Lunar iDXA machine (GE Healthcare, US), and cardiometabolic risk markers, including cholesterol, insulin, and glucose measured by 
Quest Diagnostics (Wood Dale, IL) Dietary intake was assessed using the Harvard Food Frequency Questionnaire ${ }^{10}$. Participants were also asked to wear the Actigraph GT3X triaxial accelerometer ${ }^{11}$ for 7 days to measure physical activity objectively. Functional capacity and mobility capacity were measured with the six-minute walk test ${ }^{5,12}$.

After the onset of the pandemic and associated restrictions to in-person, face-to-face human subject research interaction, we immediately began to plan how we could complete the RCT given the amount of time and effort staff and participants had contributed already as well as the scientific value of completing the study. Simultaneously, the UIC IRB developed an expedited amendment process specifically for changes to research caused by the COVID-19 shutdown. The first step in adapting to the COVID-19 research restrictions was to review and verify the options we had for remote data collection. At the outset of the pandemic, only about half of our cohort 3 participants reported having reliable online access to face-to-face remote technology (e.g., Zoom) (www.Zoom.us). Therefore, we decided to conduct telephone interviews because all of our participants had phone access. To determine the feasibility of collecting data via telephone, staff practiced and timed this data collection procedure. Based on these practice interviews, it was decided that our 14-month follow up data collection for cohort 3 could be completed over two sessions (one hour each) via telephone: one call would include lifestyle measures and dietary intake data collection, the second call would collect data using a modified cognitive assessment protocol.

Data collection was conducted while all faculty and staff were working remotely. Cell phones, hot spots, and laptops were provided to the data collection team, as needed. The study coordinator received permission to enter the offices during the shut-down to prepare all data collection material for distribution to the data collection team; once compiled, it was safely dropped off with no contact. Once our new amendment, protocol, and the addendum to informed consent forms were finalized, submitted, and approved by the IRB, data collection began. 
Most cognitive assessment measures were validated for remote collection ${ }^{13}$ and able to be administered in the same fashion as had been done during in-person, face-to-face visits. One measure, the Trail Making Test, was adapted for oral administration via telephone as previously described and validated ${ }^{14,15}$. Two of the original BRIDGE neuropsychological protocol measures, i.e., Digit Symbol Coding ${ }^{16}$ and the Stroop Color Word ${ }^{17}$ measures, were excluded because a key aspect of administration involves the exchange of test forms for participant usage and completion (i.e., reading and/or writing). During practice interviews, we developed a detailed protocol for telephone cognitive assessments.

Data collection involving specific equipment was modified wherever possible to ensure capture of these important outcomes. For example, we were able to remotely collect participant weight by mailing digital scales to each participant. Additionally, the study coordinator brought the necessary equipment for initializing, charging, and downloading accelerometers to the home of a staff member, who mailed accelerometers to cohort 3 participants and maintained contact with them via text messaging for the requisite 7 days of wear. The accelerometers were then returned to the staff member's home by US Mail. Because we were unable to meet participants in person, we did not collect blood samples, assess whole-body composition using iDXA, measure blood pressure, or assess functional capacity via the six-minute walk test.

\section{RESULTS}

As seen in Table 1, study participants were at increased risk for COVID-19 due to age and comorbid medical conditions. Specifically, the mean age was 66.4 years, and $26.2 \%$ were $\geq 70$ years. While all of the participants were obese (body mass index (BMI) $\geq 30 \mathrm{~kg} / \mathrm{m}^{2}$ ), $37.7 \%$ had class II obesity $\left(\mathrm{BMI}=35\right.$ to $\left.<40 \mathrm{~kg} / \mathrm{m}^{2}\right)$, and $27.9 \%$ had class III obesity $\left(\mathrm{BMI} \geq 40 \mathrm{~kg} / \mathrm{m}^{2}\right)$. Most participants had been diagnosed with high blood pressure (68.9\%), and many were diagnosed with high cholesterol (46.5\%) and type 2 diabetes (23.0\%). 
As shown in Table 2, participation in remote data collection by Cohort 3 at 14 months (final data collection) did not significantly differ from participation in face-to-face, in-person data collection by Cohorts 1 and 2 at 14 months. The only exception was the collection of accelerometer data, though the difference was not statistically significant $(63.9 \%$ for $\mathrm{C} 3$ vs. $77.4 \%$ for $\mathrm{C} 1+\mathrm{C} 2$, $\mathrm{p}=0.052$ ). As noted above, we could not collect blood samples, assess body composition, blood pressure, or complete the six-minute walk test.

\section{DISCUSSION}

COVID-19 will not be the last regional, national, or international disruption that affects day-today life ${ }^{18}$. Clinical research studies must have the ability to adapt to such disruptions while respecting safety and confidentiality of participants and staff. Researchers have an obligation to implement and measure the feasibility and scalability of new approaches and the impact of these new approaches on participants and communities. These data will provide the basis for further guidelines regarding how to conduct research during times of crisis ${ }^{1}$.

The health inequities in the US that impact minority communities existed prior to the COVID-19 pandemic ${ }^{19}$. In Chicago, African Americans make up 30\% of the population; yet, they represent $50 \%$ of COVID-19 cases and approximately $70 \%$ of COVID-19 deaths, most of which are concentrated in the most vulnerable communities ${ }^{20}$. Given our involvement in these communities pre pandemic and the trusting relationships we had built between staff and BRIDGE participants, there was strong motivation to complete our research work together. Cohort 3 participants had already completed the full 8-month intervention and post-intervention data collection. We clearly communicated our appreciation for the time participants were taking to provide follow-up data while living with the pandemic's ongoing day-to-day challenges. For example, we conducted wellness calls and discussed relevant non-research related issues such as COVID-19 safety precautions. In our interactions with our older adult participants, many of 
whom lived alone, most reported remaining at home and in contact with friends and family via phone and text messaging.

In retrospect, there could have been changes to data collection that may have been easier for participants. For example, many participants reported phone interview fatigue, as data collection lasted approximately two hours over two sessions. Perhaps participants could have selfadministered their dietary intake and lifestyle measures via either a postage-paid return envelope system or an online portal. We could have further explored using online platforms, but not all participants had regular online access, and at the beginning of the pandemic, there were some concern regarding maintaining confidentiality. However, since the start of the pandemic, many of these video platforms have increasingly improved their security measures. Additionally, given that most of our participants had smartphones, we could have further explored using RedCap surveys online. Nevertheless, compared to in person assessments pre-pandemic that required multiple visits to our offices and a minimum of three hours of data collection, remote assessments were likely easier for older participants. This could portend the greater use of remote assessments even after the COVID-19 pandemic ends.

The COVID-19 pandemic will continue to disrupt research activities for months to come. Researchers need to utilize existing communication platforms (e.g., text messaging, email, and remote data collection) while, first and foremost, protecting the safety of study participants and staff ${ }^{18}$. We are currently analyzing our data, having successfully collected the majority of our primary outcome cognitive data and our secondary weight, dietary intake, physical activity, and lifestyle behavior data. Given the dearth of effective pharmacological treatments to prevent or halt cognitive decline, particularly in at-risk obese African American older adults ${ }^{21-23}$, and even int the face of this global pandemic, this study has the potential to provide important insights regarding the role of lifestyle in maintaining cognitive health in aging populations ${ }^{24-26}$.

Table 1. Participant characteristics at baseline, Cohort 3 


\begin{tabular}{|c|c|c|c|}
\hline & $\mathrm{N}$ & $\begin{array}{r}\text { Mean } \\
\text { or \% }\end{array}$ & $\begin{array}{l}\text { SD } \\
\text { or N }\end{array}$ \\
\hline Age at randomization, $\mathrm{yr}$ & 61 & 66.4 & $(6.2)$ \\
\hline $55-69$ & & $73.8 \%$ & $(45)$ \\
\hline$\geq 70$ & & $26.2 \%$ & (16) \\
\hline Gender & 61 & & \\
\hline Female & & $93.4 \%$ & (57) \\
\hline Male & & $6.6 \%$ & $(4)$ \\
\hline Race & 61 & & \\
\hline Black or African-American, not Hispanic & & $91.8 \%$ & $(56)$ \\
\hline Hispanic & & $1.6 \%$ & (1) \\
\hline Native American & & $1.6 \%$ & (1) \\
\hline Multiracial & & $4.9 \%$ & (3) \\
\hline Marital status & 61 & & \\
\hline Single & & $13.1 \%$ & $(8)$ \\
\hline Married & & $36.1 \%$ & (22) \\
\hline Widowed & & $21.3 \%$ & (13) \\
\hline Divorced & & $29.5 \%$ & (18) \\
\hline Medical conditions & & & \\
\hline High blood pressure & 61 & $68.9 \%$ & $(42)$ \\
\hline High cholesterol & 61 & $47.5 \%$ & (29) \\
\hline Type 2 diabetes & 61 & $23.0 \%$ & (14) \\
\hline Sleep apnea & 61 & $24.6 \%$ & (15) \\
\hline Weight, kg & 61 & 102.1 & $(15.3)$ \\
\hline Height, cm & 61 & 164.5 & (6.8) \\
\hline BMI, kg/m² & 61 & 37.8 & (5.5) \\
\hline BMI category & 61 & & \\
\hline Obesity class I $\left(30-<35 \mathrm{~kg} / \mathrm{m}^{2}\right)$ & & $34.4 \%$ & (21) \\
\hline Obesity class II $\left(35-<40 \mathrm{~kg} / \mathrm{m}^{2}\right)$ & & $37.7 \%$ & (44) \\
\hline Obesity class III $\left(\geq 40 \mathrm{~kg} / \mathrm{m}^{2}\right)$ & & $27.9 \%$ & (17) \\
\hline Percent body fat & 60 & 48.5 & (5.1) \\
\hline
\end{tabular}




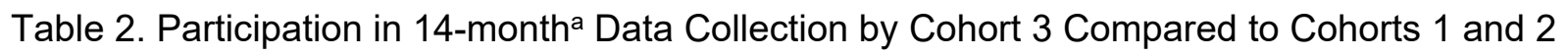

\begin{tabular}{|l|r|r|r|r|r|}
\hline & \multicolumn{2}{|c|}{$\mathrm{C} 3(\mathrm{~N}=61)$} & \multicolumn{2}{c|}{$\mathrm{C} 1+2(\mathrm{~N}=124)$} & \\
\hline & $\%$ & $\mathrm{~N}$ & $\%$ & $\mathrm{~N}$ & $\mathrm{p}^{\mathrm{b}}$ \\
\hline Any data $^{\mathrm{c}}$ & $86.9 \%$ & 53 & $87.9 \%$ & 109 & 0.84 \\
\hline Cognitive measures & $77.0 \%$ & 47 & $78.2 \%$ & 97 & 0.86 \\
\hline Diet intake & $86.9 \%$ & 53 & $87.1 \%$ & 108 & 0.97 \\
\hline Weight & $82.0 \%$ & 50 & $85.5 \%$ & 106 & 0.54 \\
\hline Lifestyle questionnaires & $86.9 \%$ & 53 & $87.9 \%$ & 109 & 0.84 \\
\hline Accelerometer & $63.9 \%$ & 39 & $77.4 \%$ & 96 & 0.052 \\
\hline
\end{tabular}

a Data collection for the 14-month visit ran from 6/2020-9/2020 for Cohort 3, from 5/2019-9/2019 for Cohort 2, and from 6/2018-10/2018 for Cohort 1.

${ }^{b}$ From chi-square tests for differences between Cohort 3 and Cohort 1+2.

c Includes only measures collected at the 14-month visit for Cohort 3: cognitive, diet, weight, questionnaires, accelerometer. 


\section{References}

1. McDermott MM, Newman AB. Preserving clinical trial integrity during the coronavirus pandemic. JAMA. 2020.

2. Coleman BC, Kean J, Brandt CA, Peduzzi P, Kerns RD. Adapting to disruption of research during the COVID-19 pandemic while testing nonpharmacological approaches to pain management.

Translational behavioral medicine. 2020;10(4):827-34.

3. Trends, Charts, and Maps: Clinicaltrials.gov; 2021 [Available from: https://clinicaltrials.gov/ct2/resources/trends.

4. WHO coronavirus disease (COVID-19) dashboard Geneva: World Health Organization; 2020 [Available from: https://covid19.who.int/

5. Tussing-Humphreys L, Lamar M, Blumenthal JA, Babyak M, Fantuzzi G, Blumstein L, et al. Building research in diet and cognition: The BRIDGE randomized controlled trial. Contemporary clinical trials. 2017;59:87-97.

6. Sanchez-Flack JC, Tussing-Humphreys L, Lamar M, Fantuzzi G, Schiffer L, Blumstein L, et al. Building Research in Diet and Cognition (BRIDGE): Baseline characteristics of older obese African American adults in a randomized controlled trial to examine the effect of the Mediterranean Diet with and without weight loss on cognitive functioning. Preventive Medicine Reports. 2020:101302.

7. COVID-19: Center for Disease Control and Prevention; [Available from: https://www.cdc.gov/coronavirus/2019-ncov/index.html.

8. Coronavirus Disease 2019 (COVID-19): Information for NIH Applicants and Recipients of NIH Funding: National Institutes of Health; [Available from: https://grants.nih.gov/grants/natural disasters/corona-virus.htm.

9. Hachinski V, ladecola C, Petersen RC, Breteler MM, Nyenhuis DL, Black SE, et al. National Institute of Neurological Disorders and Stroke-Canadian stroke network vascular cognitive impairment harmonization standards. Stroke. 2006;37(9):2220-41.

10. Willett WC, Sampson L, Stampfer MJ, Rosner B, Bain C, Witschi J, et al. Reproducibility and validity of a semiquantitative food frequency questionnaire. American journal of epidemiology. 1985;122(1):51-65.

11. Santos-Lozano A, Marín PJ, Torres-Luque G, Ruiz JR, Lucía A, Garatachea N. Technical variability of the GT3X accelerometer. Medical engineering \& physics. 2012;34(6):787-90.

12. Laboratories ACoPSfCPF. ATS statement: guidelines for the six-minute walk test. Am J Respir Crit Care Med. 2002;166:111-7.

13. Wilson RS, Leurgans SE, Foroud TM, Sweet RA, Graff-Radford N, Mayeux R, et al. Telephone assessment of cognitive function in the late-onset Alzheimer's disease family study. Archives of neurology. 2010;67(7):855-61.

14. Ricker JH, Axelrod BN. Analysis of an oral paradigm for the Trail Making Test. Assessment. 1994;1(1):47-51.

15. Kaemmerer T, Riordan P. Oral adaptation of the trail making test: A practical review. Applied Neuropsychology: Adult. 2016;23(5):384-9.

16. Wechsler D. Wechsler Memory Scale. 3rd ed. San Antonio, TX: Psychological Corporation; 1997c. 17. Stroop JR. Studies of interference in serial verbal reactions. Journal of experimental psychology. 1935;18(6):643.

18. Singh JA, Bandewar SV, Bukusi EA. The impact of the COVID-19 pandemic response on other health research. Bulletin of the World Health Organization. 2020;98(9):625.

19. Alcendor DJ. Racial disparities-associated COVID-19 mortality among minority populations in the US. Journal of clinical medicine. 2020;9(8):2442. 
20. Reyes C, Husain N, Gutowski C, St Clair S, Pratt G. Chicago's coronavirus disparity: black Chicagoans are dying at nearly six times the rate of white residents, data show. Chicago Tribune. 2020 April 7, 2020.

21. Shadlen MF, Siscovick D, Fitzpatrick AL, Dulberg C, Kuller LH, Jackson S. Education, cognitive test scores, and black-white differences in dementia risk. Journal of the American Geriatrics Society. 2006;54(6):898-905.

22. Mehta KM, Simonsick EM, Rooks R, Newman AB, Pope SK, Rubin SM, et al. Black and white differences in cognitive function test scores: what explains the difference? Journal of the American Geriatrics Society. 2004;52(12):2120-7.

23. Rovner BW, Casten RJ, Harris LF. Cultural diversity and views on Alzheimer's disease in older African Americans. Alzheimer disease and associated disorders. 2013;27(2):133.

24. Lourida I, Soni M, Thompson-Coon J, Purandare N, Lang IA, Ukoumunne OC, et al.

Mediterranean diet, cognitive function, and dementia: a systematic review. Epidemiology. 2013:479-89.

25. Valls-Pedret C, Ros E. Commentary: Mediterranean Diet and Cognitive OutcomesEpidemiological Evidence Suggestive, Randomized Trials Needed. Epidemiology. 2013;24(4):503-6.

26. Rolland $Y$, van Kan GA, Vellas B. Healthy brain aging: role of exercise and physical activity. Clinics in geriatric medicine. 2010;26(1):75-87. 